\title{
Los documentos de voluntades anticipadas
}

\section{The living will}

\author{
K. Martínez
}

\section{RESUMEN}

La importancia de una toma informada y compartida de decisiones entre el paciente y el profesional de salud que le atiende es fundamental para la calidad de la asistencia y los resultados de ésta. Los documentos de voluntades anticipadas no son más que una concreción de esta realidad. La aceptación legal de los mismos ha supuesto un espaldarazo al respeto por la autonomía de los enfermos. Sin embargo, las leyes aprobadas presentan algunas incongruencias y la práctica sanitaria relacionada con dichos documentos adolece de múltiples problemas éticos prácticos sobre los que se invita a reflexionar. Se concluye recomendando la adopción de medidas encaminadas a fortalecer la planificación anticipada de la atención, cuestión para la que se deberán dar cambios profundos en la formación de los profesionales y en las políticas institucionales sanitarias.

Palabras clave. Voluntades anticipadas. Instrucciones previas. Testamento vital. Planificación anticipada de la atención. Bioética.

\begin{abstract}
The importance of decision making that is informed and shared between the patient and the health professional caring for him/her is essential for the quality of care and its results. Living wills are no more than a concrete expression of this reality. Their legal acceptance has meant recognition of respect for the autonomy of patients. However, the laws passed contain some incongruities and the health practice related to these documents suffers from numerous practical and ethical problems on which reflection is invited. The article concludes by recommending the adoption of measures aimed at strengthening the advance planning of health care, a question that requires profound changes in the training of professionals and in the policies of the health institutions.
\end{abstract}

Key words. Living wills. Prior instructions. Advance planning of health care. Bioethics.

An. Sist. Sanit. Navar. 2007; 30 (Supl. 3): 87-102.

Médico Intensivista. Hospital de Navarra. Magíster en Bioética.

\author{
Correspondencia: \\ Medicina Intensiva \\ Hospital de Navarra \\ Irunlarrea, 3 \\ 31007 Pamplona \\ e-mail: kmartinez@cfnavarra.es
}




\section{IMPORTANCIA DE LA TOMA INFORMADA, DELIBERADA Y CONSENSUADA DE LAS DECISIONES EN SALUD}

La relación clínica es un proceso dinámico y complejo cuyo curso no puede ser predicho de antemano porque no existen dos relaciones clínicas iguales ${ }^{1}$. A pesar de que una gran mayoría de pacientes parece desear ser informada sobre sus enfermedades, existe entre los mismos una considerable variedad individual sobre su deseo de participar en la toma de decisiones, y es imposible predecir las preferencias de un paciente concreto con relación a sus deseos de participación activa en la toma de decisiones ${ }^{2}$. Está demostrado que la participación de los pacientes conduce a mejores resultados de salud lo que coincide con la antigua creencia de que, ya se trate del mantenimiento de la salud o del tratamiento de una persona enferma, la base del buen cuidado es una relación cálida y confortable entre médico y paciente ${ }^{3}$. Es también sabido que las actitudes distantes por parte de los médicos provocan falta de adhesión a los tratamientos médicos, fallos en la comunicación, insatisfacción tanto entre los pacientes como entre los sanitarios, denuncias por mala praxis, y "pérdida de fe" en la medicina ${ }^{4}$. Cuando surgen desacuerdos entre paciente y médico, puede ocurrir que el médico se centre en lograr metas no deseadas por el paciente, y al revés, puede también darse que éste vaya a la búsqueda de algo considerado como imposible por el médico. El desacuerdo entre profesionales de la salud y pacientes puede alargar la intervención médica indefinidamente, cambiar el proceso natural del morir, y provocar confusión moral en los profesionales, el enfermo y su familia.

Al final de la vida, algunos médicos, guiados por el imperativo tecnológico, son partidarios de poner todos los tratamientos y de hacer todos los esfuerzos posibles a su alcance, provocando cierta obstinación terapéutica en contra de los intereses o deseos del paciente, incluso; otros no saben cómo manejar la incertidumbre que se halla en la base de la medicina más que mediante el dogmatismo y el activismo clí- nicos. Parece lógico pensar que las decisiones deberían tomarse basándose en el conocimiento y en la comprensión de los datos médicos, los pronósticos de la enfermedad y los objetivos negociados entre paciente y profesional, y no bajo el influjo de la enfermedad aguda o bajo el miedo o con dolor. Forzar a los pacientes en estas circunstancias a decir qué tratamientos prefieren o cuáles no, o a nombrar un sustituto que decida por ellos, quizás sea legalmente aceptable, pero éticamente es cuando menos problemático ${ }^{5-7}$.

\section{LOS DOCUMENTOS DE VOLUNTADES ANTICIPADAS}

Hace algo más de tres décadas, el abogado Louis Kutner propuso los "living will" como medio de resolución de los conflictos que surgen al final de la vida entre médicos y pacientes en relación con las preferencias de éstos por unos tratamientos, otros o ninguno ${ }^{8}$. El llamado testamento vital, término mal traducido aunque popularmente aceptado, que en las leyes españolas y de las Comunidades Autónomas ha pasado a denominarse de formas varias -instrucciones previas, voluntades anticipadas, manifestaciones anticipadas de voluntad, expresión anticipada de voluntades o voluntades vitales anticipadas- (aunque para evitar la diversificación utilizaremos las siglas DVA para referirnos a todos ellos), sería así el documento que muestra cómo deseamos ser tratados en un momento en que, en caso de incapacidad debida a enfermedad, lesión o edad, no podamos expresar nuestros deseos. Teóricamente, el DVA permite elegir toda la intervención posible, poner ciertos límites a la misma o rechazarla totalmente.

Los DVA adquirieron su esplendor tras la aprobación de la llamada "Patient SelfDetermination Act" -Ley de Autodeterminación del Paciente (PSDA)- en los Estados Unidos (EEUU), a raíz del sonado y publicitado caso de Nancy Cruzan ${ }^{9}$. El Tribunal Supremo de Justicia de EEUU reconoció en Noviembre de 1990 el derecho de los pacientes competentes a rechazar un tratamiento médico no deseado y fijó un procedimiento para la toma de decisiones por parte de los sustitutos o representantes de 
los pacientes no competentes ${ }^{10}$. Posteriormente el Tribunal de Missouri explicitaba la necesidad de que hubiera una "evidencia clara y convincente" del deseo del paciente de no ser tratado. Desde entonces en los EEUU todos los hospitales, residencias de la tercera edad, etc., tienen la obligación legal de desarrollar directrices escritas en relación con los DVA y de educar a los profesionales sanitarios (PS) sobre la cuestión, así como de preguntar a los pacientes en el momento del ingreso si han redactado su DVA y de informarles del derecho que tienen a hacerlo ${ }^{11}$.

Aprobada la PSDA en un momento de publicidad de un caso notorio de obstinación terapéutica, los Tribunales de Justicia suministraron una vía legal para facilitar la toma de decisiones en estas situaciones. Se creía y esperaba entonces que los DVA protegerían las elecciones autónomas de los pacientes, facilitarían la comunicación entre médico y paciente para prevenir el encarnizamiento terapéutico y permitirían, además, ahorrar dinero ${ }^{12-14}$. Por todo ello fue apoyada casi universalmente por los bioeticistas, que vieron en ella un instrumento que serviría para proteger la autonomía de los pacientes; por los pacientes, que pensaron que a través de los DVA podrían verse libres de la imposición médica de tratamientos interminables y sin sentido; por los médicos, que vieron en ellos una herramienta a través de la cual les sería posible conocer los deseos de los pacientes, lo que les facilitaría la toma de decisiones en situaciones comprometidas y difíciles; y por los gestores, que pensaron que todo ello contribuiría a disminuir los costes de la asistencia sani$\operatorname{taria}^{15-17}$. Pero también se levantaron voces críticas que mostraron su preocupación ${ }^{18-20}$. Entre ellas, es famosa la carta de 16 eticistas, médicos, enfermeros y abogados en la que afirmaban que los pacientes prefieren no hablar de la muerte y de la discapacidad, que no suelen predecir con claridad sus deseos de cara al futuro, que las opiniones cambian, y que el representante no es siempre el mejor traductor de los intereses del paciente ${ }^{21}$. Algunos, por lo tanto, pronto vieron que las obligaciones superficiales de la PSDA eran fáciles de cumplir pero que para cumplir las fundamentales eran necesarios cambios de fondo en la política institucional sanitaria y en la educación de los PS y de la sociedad.

\section{UNA BREVE REFERENCIA A LA LEGISLACIÓN ESPAÑOLA}

En España, la ratificación y entrada en vigor en enero de 2000 del Convenio para la Protección de los Derechos Humanos y la Dignidad del Ser Humano con respecto a las Aplicaciones de la Biología y la Medici$n^{22}$, más conocido como Convenio de Oviedo, supusieron el punto de partida para el reconocimiento normativo de las DVA. El artículo 9 de dicho Convenio dice que: "Serán tomados en consideración los deseos expresados anteriormente con respecto a una intervención médica por un paciente que, en el momento de la intervención, no se encuentre en situación de poder expresar su voluntad".

La ratificación del Convenio obligó a modificar algunos aspectos de la Ley General de Sanidad (LGS) y fruto de dichos cambios es la Ley 41/2002, de 14 de noviembre, básica reguladora de la autonomía del paciente y de derechos y obligaciones en materia de información y documentación clínica $^{23}$. Simón y Barrio $^{24}$ subrayan la importante solidez jurídica con que tanto el Convenio de Oviedo como la Ley 41/2002 dotan al desarrollo de las decisiones de representación y a la introducción de los DVA en España, tras mostrar ciertas reticencias sobre algunos aspectos de la LGS, tales como la denominación "familiares o personas a él (el enfermo) allegadas" por resultar ambiguas a la hora de decidir quién podía ostentar el derecho de representación cuando el paciente no estuviera capacitado para la toma decisiones.

La Ley 41/2002 en su artículo 11, punto 1 , especifica que "Por el documento de instrucciones previas, una persona mayor de edad, capaz y libre, manifiesta anticipadamente su voluntad, con objeto de que ésta se cumpla en el momento en que llegue a situaciones en cuyas circunstancias no sea capaz de expresarlos personalmente, sobre los cuidados y el tratamiento de su salud o, una vez llegado el fallecimiento, sobre el destino de su cuerpo o de los órganos del mismo. El otorgante del documento puede designar, 
además, un representante para que, llegado el caso, sirva como interlocutor suyo con el médico o el equipo sanitario para procurar el cumplimiento de las instrucciones previas".

Distintas comunidades autónomas han promulgado, algunas previamente a la Ley $41 / 2002$, otras posteriormente, leyes que hacen referencia a este derecho, presentando algunas importantes diferencias entre sí (en cuanto a denominación, concepto, contenido, requisitos para el otorgamiento, formalización, eficacia, etc.... $)^{25}$ que deben dar origen a una reflexión legal al respecto $\mathrm{y}$, seguramente, a posibles modificaciones de dichos textos.

Seoane $^{26}$, por su parte, escribe que "Junto a leyes o decretos resultado de un proceso de madurez y de deliberación social, profesional y política o parlamentaria, que representan instrumentos valiosos para orientar los procesos de toma de decisiones, hallamos normas precipitadas, imprecisas y confusas e incluso contradictorias".

No entraré a analizar los contenidos, los límites y las aplicaciones clínicas de los DVA por haber sido ya extensamente publicitados en la bibliografía española ${ }^{27}$ y porque considero más importante en este momento continuar haciendo un análisis crítico de los fundamentos éticos y de los problemas prácticos de los DVA. Sí quiero recordar que, a pesar de que la mayoría de las Comunidades Autónomas, en consonancia con sus propias leyes, han editado guías de información sobre los DVA para los profesionales y los ciudadanos, todo lo relacionado con los mismos sigue siendo un tema muy desconocido tanto por los profesionales de la salud ${ }^{28,29}$ como por la propia ciudadanía ${ }^{30-32}$.

\section{REFLEXIONES SOBRE ALGUNAS CUESTIONES ÉTICAS (Y POR TANTO PRÁCTICAS)}

Afirmo, de entrada, que los DVA no están libres de riesgos. Si se interpretan o se redactan mal, pueden llevar a que el médico trate menos de lo debido al paciente; pueden añadir una capa técnica más al proceso ya muy técnico y complicado de toma de decisiones; pueden ser utilizados como un instrumento para disminuir la responsabilidad que en estas cuestiones tienen los profesionales y las instituciones sanitarias; y también, finalmente, pueden llevar a los sanitarios a sentirse libres de la carga de tener que preocuparse de sus pacientes. Porque, aunque los DVA surgieron para que médicos y pacientes se comunicaran de forma permanente, al refugiarse en una regla o norma general, los médicos pueden utilizarlos para escapar y alejarse fácilmente de la preocupación por la situación particular del paciente concreto, dejando al enfermo abandonado. En mi opinión, los pacientes que rellenan sin más los documentos que se les ofrecen son pacientes abandonados porque, a veces, debido a la falta de asesoramiento profesional, pueden llegar a formular instrucciones mutuamente inconsistentes ${ }^{33}$. Del mismo modo que también son pacientes abandonados quienes firman los documentos de consentimiento informado sin haber sido debidamente informados y sin haber sido guiados a través de la información mediante una comunicación respetuosa y empática. Para ello se necesita lo que Epstein ${ }^{34}$ llama una "práctica juiciosa", un modo de hacer caracterizado por la observación activa de uno mismo, del paciente y del problema a resolver; la visión periférica; el proceso de preatención; la curiosidad crítica; la valentía de ver el mundo tal cual es más que como uno desearía que fuera; la voluntad de examinar y de abandonar categorías y prejuicios; la adopción de la mente de un principiante; la humildad para tolerar la existencia de áreas de incompetencia en uno mismo; la conexión entre el sujeto que conoce y el que es conocido; la compasión basada en la reflexión interior, y la presencia. Esta práctica juiciosa lleva tanto al médico como al paciente a una comprensión integrada de la enfermedad y de la experiencia del enfermar facilitadora de una toma compartida de decisiones. También de cara al final de la vida. Y a pesar de ello, cabe recordar que los pacientes reinterpretan siempre la información recibida y que ni siquiera la experiencia con la enfermedad es un buen maestro, no habiéndose encontrado asociaciones significativas entre dicha experiencia con la formulación de documentos de voluntades anticipadas 
(DVA) ni con el conocimiento de las opciones existentes al final de la vida ${ }^{35-37}$.

Una gran evidencia indica que las respuestas a las preguntas dependen de cómo se hacen éstas, y en esa medida, las preferencias sobre tratamientos están influidas por factores como la utilización o no de tasas de éxito o de fracaso, el nivel de detalle utilizado, y con si se explican de entrada las consecuencias a largo plazo ${ }^{38-40}$.

No se trata sólo, creo yo, de que las personas formalicen DVA, tal y como afirman y pretender mejorar algunos autores $^{41}$, sino de que lo hagan bien. Y es que elaborar un DVA sin una deliberación entre médico y paciente difícilmente es mejor que no tener un DVA. Son muchos los autores que afirman que esta comunicación entre paciente y personal sanitario es muy deficiente incluso a la hora de redactar los $\mathrm{DVA}^{42-45}$. Además, si lo que se pretende con los DVA es proteger la autonomía del paciente, no me cabe la menor duda de que esto se consigue mejor en la consulta de un médico comprometido con su paciente que en el despacho de un notario o en la oficina de un funcionario municipal, porque, como dice Linda Emanuel ${ }^{46}$, los DVA ayudan a los pacientes y a sus médicos a hablar del morir y pueden por tanto "abrir la puerta a una aproximación positiva y cuidadosa a la muerte".

Preocupado por estas cuestiones, Welie $^{47}$ aporta algunas reflexiones éticas sobre los DVA, reflexiones que Seoane transporta al ámbito de la ley en el artículo antes citado para elaborar unas propuestas de cara a mejorar la regulación jurídica de los mismos ${ }^{38}$. Unas hacen referencia al peso moral de la autonomía del paciente en la toma de decisiones y por ello se pregunta si la autonomía es siempre el principio determinante a la hora de tomar decisiones sanitarias, afirmando que en un contexto liberal, la autonomía es colocada generalmente por encima de la beneficencia, pero que sería éticamente inconsistente si aceptáramos que la libertad de elección es el único valor, o el más importante, para los pacientes. Los pacientes no buscan la libertad que los políticos liberales debaten; buscan respeto como personas, como individuos con una vida única, una única historia, una biografía propia y unos objetivos únicos. Respetar genuinamente a las personas no quiere decir abandonarlas a su libertad sino cuidarlas y ayudarlas de forma que esta actitud les beneficie en su propia especificidad. Y afirma que quizás el mayor peligro para la atención a la salud en el siglo XXI no sea ya el paternalismo autocrático de los médicos sino el abandono respetuoso de los pacientes. Por eso se cuestiona si pueden ponerse límites a la autonomía del paciente. Y responde afirmando que el principio del respeto al derecho de autodeterminación tiene dos fundamentos morales: Por un lado, el derecho "a ser dejado en paz"; y por otro, el derecho a decidir qué es bueno para uno mismo. El primer fundamento se traduce en una obligación "negativa" por parte del cuidador, esto es, en la obligación de abstenerse de realizar intervenciones médicas no consentidas. El segundo implica una obligación "positiva" mucho más fuerte, la de cumplir los deseos del paciente de cara al tratamiento. Ambas fundamentaciones morales implican el deber por parte del cuidador de aceptar el rechazo del paciente a determinadas intervenciones médicas, incluidos los tratamientos de soporte vital; pero sólo la segunda implicaría la obligación moral de ejecutar una intervención médica concreta a petición del paciente. Estas diferencias en la naturaleza y en la amplitud de las dos fundamentaciones morales del deber de respetar los deseos autónomos del paciente tienen lógicamente consecuencias en relación con la obligatoriedad de los DVA y de los juicios por sustitución, sustitutivos o mediante representante.

Un DVA genuino no sólo expresa las preferencias, valores o intereses personales de un paciente sino que es también, de alguna forma, un documento ejecutivo. Pero el valor ejecutivo de una directriz que rechaza un tratamiento es siempre mayor que el de la que lo solicita. O más concretamente: la obligación correspondiente por parte de los cuidadores de seguir una directriz es mayor cuando los pacientes rechazan un tratamiento. Ningún médico está obligado a proporcionar tratamiento que él sabe es perjudicial para el paciente, 
pero sí tiene que aceptar el rechazo del paciente a un tratamiento aunque pueda ser beneficioso para el enfermo. Un rechazo de tratamiento de un paciente competente ha de ser respetado siempre, pero, ¿es la obligación del médico de aceptar tal rechazo igualmente fuerte cuando tal voluntad viene reflejada a través de un DVA?.

Hay dudas sobre el grado de autoridad de las personas para tomar decisiones para un "futuro yo". Si aceptamos, tal y como defiende Parfitt ${ }^{48}$, que la continuidad y la conexión psicológicas durante la vida humana pueden disminuir, también se puede debilitar la unión moral de las obligaciones que emanan de un DVA con la documentación escrita en un estadio previo de la vida. Si Parfitt estuviera en lo cierto, el sentido último de los DVA queda restringido, ya que la primera de estas personas no podría determinar a priori decisiones moralmente obligatorias en lo referente a tratamientos para una ulterior "segunda" persona ${ }^{49}$. Al contrario, otras personas (familiares, amigos, médicos o enfermeras) que han conocido a la "nueva" persona estarían moralmente más justificadas para tomar decisiones médicas en su lugar, porque la "nueva" persona podría tener intereses diferentes a los de la "antigua" personalidad ${ }^{50}$. Pero considero que esta teoría es rechazable porque el derecho de respeto a la autodeterminación debe ser entendido como el derecho de una y la misma persona, que como persona completa ha vivido a través de varios estadios de vida y puede hacer previsiones para estadios futuros. Aunque conviene no olvidar que con el paso del tiempo los DVA pueden de hecho traicionar los auténticos deseos de los pacientes ${ }^{51,52}$.

Existe así mismo cierto grado de preocupación por el grado de autenticidad y estabilidad de la decisión, dado que el DVA de un paciente generalmente está basado en la reconstrucción imaginaria del mismo sobre cómo sería su vida en la nunca antes experimentada condición X. Tal reconstrucción imaginaria es altamente problemática por la dificultad que todos tenemos para predecir hechos futuros, porque un paciente raramente puede prever la naturaleza exacta de su condi- ción futura adecuadamente, y por la dificultad de predecir no ya los hechos futuros sino las apreciaciones que en el futuro una persona tendrá de esos hechos, y existe de hecho una amplísima bibliografía que da cuenta de los cambios de los deseos de las personas, incluso para cosas muy sencillas, a lo largo del tiem$\mathrm{po}^{53-55}$. Si de todos es conocida la dificultad de la toma de decisiones sanitarias, incluso para problemas que se dan en ese mismo momento, debida a la incertidumbre que las rodea, ¿cuánto más difícil no será decidir para un futuro hipotético ${ }^{56}$.

Hay una preocupación ética importante relacionada con la existencia de salvaguardas apropiadas para la toma de decisiones. Éstas hacen referencia al grado de obligatoriedad de las preferencias y a cuándo podrían ser desoídas. Como justificaciones para no seguir los DVA se suelen citar: la calidad del DVA es muy baja; la información accesible al profesional contradice al DVA; la invasividad de la intervención es pequeña comparada con las consecuencias derivadas de no realizarla; y finalmente, en el caso de un paciente que rechaza un tratamiento, el nivel o grado en el que la imposición del tratamiento sería percibida por el paciente como una violación de su voluntad. Son interesantes al respecto el caso de Texas que ha legislado la forma en que los médicos pueden oponerse a las voluntades del paciente cuando éste ha solicitado tratamientos considerados inefectivos o inapropiados por los profesionales sanitarios ${ }^{57,58}$ así como las críticas recibidas por la utilitarista revisión hecha en 2006 de la legislación americana con la intención de permitir el no seguimiento de los DVA de cara a conseguir órganos para trasplante ${ }^{59}$.

Cabe recordar, además, que toda información que no parte directamente del propio paciente es esencialmente poco fiable. Mientras que uno puede tener en cuenta los documentos escritos por el paciente o las narraciones grabadas y corroboradas por él mismo, ningún análisis interpretado o, aún peor, intuitivo por familiares, amigos y otras terceras personas de los valores personales e intereses privados del paciente es aceptable sin más deliberación. La vida es lo suficientemente impre- 
decible como para ser adecuadamente fijada en los DVA. Pero sin una evidencia carente de ambigüedad, el representante no puede reconstruir lo que el paciente hubiera deseado si no se hubiera vuelto incompetente. Idealmente, por tanto, los sustitutos no debieran descansar sólo en testimonios pasados del y sobre el paciente sino que debieran participar directa y activamente en las deliberaciones previas a la redacción del DVA para poder interpretar adecuadamente las palabras y los deseos del enfermo al que sustituyen o complementan en la toma de decisiones.

También es clara la preocupación sobre la validez en el tiempo de un DVA. ¿Tiene la misma validez y, por tanto, obliga de igual manera un DVA redactado hace cinco días que uno escrito hace cinco meses o cinco años?

Finalmente, se debe llamar la atención sobre el diferente valor de la autonomía en los diversos grupos étnicos y comunidades culturales, aspecto muy a tener en cuenta en nuestra sociedad cada vez más multicultural.

\section{LA IMPORTANCIA DE LAS CUESTIONES PRÁCTICAS (Y POR TANTO ÉTICAS)}

Hay por todo ello quien se pregunta si realmente merece la pena elaborar un DVA. En 1991, Brock ${ }^{60}$ especificaba varias situaciones que obligaban al médico a no hacer caso a lo expuesto en el DVA. Esto, en su opinión, afirmaba el valor de los DVA en casi todos los demás casos. Personalmente pienso que cada DVA debe ser analizado y valorado en cada situación concreta porque la existencia de un DVA no exime al médico de la obligación de analizar qué es lo más conveniente para los intereses de su paciente en cada momento. Cada DVA debe ser valorado individualmente; no se puede aceptar su contenido sin más. Esta postura, en alguna medida, casi hace al DVA un documento éticamente superfluo para los médicos porque, en el fondo, los médicos deberán hablar y deliberar igualmente con los representantes de un paciente que tiene un DVA que con los del que no lo tiene para intentar tomar la decisión más acorde a sus intereses y valores actuales. Porque el hecho de que el DVA sea un documento legal no lo hace ni legítimo ni más válido éticamente que una buena deliberación.

A pesar de las normas y obligaciones que menciona la PSDA un porcentaje muy pequeño de la población americana ha redactado DVA. Esta baja incidencia es achacada a que los profesionales de la salud (PS) y las instituciones sanitarias creen que esta labor es tarea exclusiva de los pacientes; a que es incómodo para los PS hablar de cuestiones relacionadas con la muerte; a que es difícil hacerlos; a que muchos dudan que lo necesiten; a que no creen que ello afecte al tratamiento que vayan a recibir; a que es incompatible con sus tradiciones e ideas culturales; y a que tanto los PS como los propios ciudadanos piensan que este tema va dirigido fundamentalmente a los ancianos o enfermos crónicos ${ }^{61-63}$.

En este sentido cabe señalar que cuando los pacientes que acuden a un centro de salud son informados sobre los DVA las personas muestran interés por los mismos y expresan su intención favorable a cumplimentarlos ${ }^{64}$, y que el número de DVA $^{65}$ y la satisfacción con la atención recibida ${ }^{66}$ aumentan.

Como se ha mencionado, los DVA ayudan a pensar en la enfermedad y en la muerte, a hablar de los deseos personales sobre estas situaciones con el médico y los seres queridos, a hablar del miedo a quedar atrapado en las redes del sistema sanitario, a aclarar preferencias sobre tratamientos, a nombrar un sustituto, etc. Pero todo ello es imposible de hacer si los pacientes, los representantes y los PS no hablan entre ellos, y si éstos no les informan y les aconsejan adecuadamente ${ }^{67}$. Y es que entender los DVA como una especie de lista de aprobación o de rechazo de determinados tratamientos no responde a las necesidades reales de los enfermos.

Jezewsi y $\mathrm{col}^{68}$ han analizado la efectividad de las intervenciones realizadas en EEUU para aumentar el cumplimiento de las obligaciones derivadas de la PSDA. Los autores encuentran que se han realizado dos tipos de intervenciones para intentar conseguir aumentar el número de DVA: didácticas e interactivas. En las primeras 
la información se proporciona a través de programas educativos, en los centros asistenciales o por correo. En las interactivas se hace mediante diálogos entre el personal sanitario y la persona concreta, que puede así hacer preguntar, aclarar dudas y solicitar ayuda para rellenar dichos documentos. El resultado del análisis de la literatura publicada no deja lugar a dudas: la estrategia didáctica no produce un aumento significativo de DVA mientras que la estrategia interactiva sí lo hace, en cantidad y en calidad. Por cierto, los resultados son mejores en todos los grupos de edad pero fundamentalmente entre la población de la tercera edad. Los autores concluyen afirmando la importancia de establecer asesorías sanitarias que garanticen que las personas que rellenan DVA lo hacen tras aclarar sus dudas y sabiendo a quién se dirigen. Sin embargo, la adición de una cinta de video a los folletos explicativos que se enviaban por correo no consiguió aumentar la prevalencia de DVA en una población de personas de la tercera edad sino que, al contrario, los pacientes se volvían más conscientes de las razones para no formalizar DVA ${ }^{69}$.

En Cataluña, al menos hasta 2003, la mayoría de DVA redactados son de personas sanas que no padecen enfermedades que pongan en grave riesgo su vida en ese momento, por lo que la ayuda personalizada de un sanitario parece muy interesante de cara a la calidad de dichos DVA ${ }^{70,71}$. Pero esto requiere de un proceso de reflexión individual y comunitaria en el que el papel de los PS es fundamental ${ }^{72}$. Porque si algo está claro en relación con los DVA y la planificación anticipada de la asistencia es que hay que sacarlos del mundo de lo legal para reinsertarlos en lo ético y profesional sanitario ${ }^{73}$. Cabe aquí resaltar el importante papel que pueden desempeñar en esta labor el personal de enfermería y otros sanitarios ${ }^{7476}$.

Por eso, si los PS y los pacientes no entramos en reflexiones más profundas a la hora de redactarlos, como hasta el momento, los DVA serán, sobre todo, una solución legal centrada en el médico, en el abogado y en intervenciones específicas distintas de las preocupaciones reales del paciente $^{77}$. Y quizás cabe también reseñar que hay quien afirma que la problemática de la implementación de los DVA no está relacionada únicamente con los problemas prácticos de los mismos (redacción, registro, etc.) sino en la dificultad de enfrentarnos a la ambivalencia que todos y cada uno de nosotros sentimos ante el problema de la muerte, de la muerte en general y de la propia en concreto $^{78}$.

\section{LA PLANIFICACIÓN ANTICIPADA DE LA ATENCIÓN}

Por todo ello se afirma que el DVA no es más que uno de los componentes de la planificación anticipada de la atención (PA). Y se define ésta como el proceso por el cual un paciente, tras deliberación con su médico, seres queridos, etc., toma decisiones sobre la atención sanitaria que desea en el futuro $^{79}$. Otros afirman que no se trata sólo de la preparación de documentos legales sino también de la deliberación con familiares y médicos sobre lo que el futuro puede ofrecer a los enfermos graves, sobre cómo quieren los pacientes y sus familias que sus creencias y preferencias guíen las decisiones (cuando aparezcan problemas médicos críticos inesperados y bruscos), y sobre cómo se podrían aliviar preocupaciones relacionadas con la situación económica, cuestiones familiares, espirituales y otras que preocupan gravemente a los pacientes moribundos y sus familias, siendo conscientes de las enormes dificultades que todo esto conlleva y que son las que quizás transformen la medicina de una mera ocupación en una verdadera profesión ${ }^{80}$. Por eso se dice que la PA es el proceso de planificación de la atención médica en el futuro, en el caso de que el paciente no sea entonces capaz de tomar sus propias decisiones; o sea, el proceso de discusión y documentación estructurada dentro del proceso habitual de atención a la salud, que se revisa y pone al día periódicamente ${ }^{81}$.

La PA tiene un objetivo global que es el de asegurar que la atención sanitaria sea conforme a las preferencias del paciente cuando éste sea incapaz de participar en la toma de decisiones, y los siguientes objetivos específicos:

1. La mejora del proceso de toma de decisiones, facilitando la toma compartida de decisiones entre pacien- 
tes, sustitutos y PS, basados en el pronóstico y las preferencias del paciente; permitiendo, sin imposiciones, la delegación de las decisiones sobre tratamientos; balanceando la especificidad con la flexibilidad en situaciones clínicas cambiantes; favoreciendo la educación pública y privada sobre el proceso del morir y cuestiones relacionadas con el mismo; y

2. La mejora de los resultados para los pacientes, optimizando el bienestar del enfermo; disminuyendo la frecuencia y la magnitud de los posibles tratamientos excesivos o excesivamente parcos; asegurando que los pacientes tienen control sobre su asistencia sanitaria; y disminuyendo las preocupaciones de las personas sobre la creación de posibles cargas a sus familiares y seres queridos ${ }^{82}$.

La PA puede objetivarse formalmente mediante documentos legales tales como el DVA, las directrices médicas o mediante la designación de un sustituto, o bien informalmente mediante la llamada "historia de valores" o mediante instrucciones verbales, recolecciones de conversaciones, anotaciones en la historia clínica, cartas, etc. Ejemplos de DVA son el de la Asociación Derecho a Morir Dignamente o el de la Conferencia Episcopal. Como ejemplo de directrices médicas se puede citar el rechazo a las transfusiones de los Testigos de Jehová. La historia de valores es un documento que proporciona información de fondo que aclara la intención de las voluntades anticipadas e incluye detalles morales, sociales, religiosos y filosóficos así como experiencias del paciente y diagnósticos médicos del mismo ${ }^{83-85}$. No se ha comprobado hasta el momento que los pacientes prefieran un tipo de DVA sobre otro ni que ninguno de ellos sea un factor suficiente para que sobre él se inicie una conversación relacionada con el tema con el médico ${ }^{86}$.

Considero que cuando una persona quiera elaborar un DVA debe al menos hacerse las siguientes cinco preguntas e intentar responderlas ${ }^{87}$ : ¿Qué quiero hacer, un DVA o nombrar a mi representante?
¿Directrices médicas o representante? ¿Directrices detalladas o generales? ¿Centradas en valores, objetivos o preferencias? ¿Directriz genérica o específica para cada enfermedad?.

En mi opinión, un buen DVA, para serlo, debe contener al menos: historia de valores, especificación de límites al tratamiento y nombramiento del sustituto (aceptado por el propio sustituto). Puede además completarse con algunas indicaciones para después del fallecimiento como puede ser el deseo de donar los órganos. Los DVA, ya lo he dicho, pueden carecer de sentido sin una comunicación abierta y honesta entre paciente y PS sobre la situación clínica del enfermo, los posibles resultados y las opciones de tratamiento ${ }^{88}$. Las decisiones son necesarias pero como integrantes de una más amplia deliberación con los pacientes sobre la base de sus valores y de su experiencia de la enferme$\operatorname{dad}^{89,90}$

Un problema añadido se deriva de que las preferencias pueden cambiar con las circunstancias $^{91,92}$. De hecho se ha comprobado que el $70 \%$ de los pacientes mantiene lo afirmado previamente y el $30 \%$ lo modifica. Aunque son más cambiantes las opiniones de quienes no han completado un DVA que las de los que sí lo han hecho. Y son más estables en el tiempo las opiniones de quienes deseaban menos tratamientos que las de los deseaban más. Cuando se producen cambios en las preferencias, generalmente van en la dirección de desear menos tratamientos. Las decisiones más sostenidas son las que se han tomado tras deliberar con el médico. En un estudio, las preferencias sobre tratamientos de soporte vital eran sólo moderadamente estables a los 2 años, y variaban según el escenario de la enfermedad. Los participantes, sin embargo, no eran conscientes de estos cambios ${ }^{93}$.

En un estudio con 201 pacientes de la tercera edad se vio que según el modo de presentación de una intervención médica, un $12 \%$ la aceptaba y un $30 \%$ la rechazaba. Tras una nueva presentación en la que los médicos les explicaron dicha intervención de forma distinta, el $75 \%$ de los enfermos cambió su respuesta. Lo que no hace más 
que subrayar la importancia de hablar siempre con el paciente ${ }^{94,95}$. Pero es que, además, se ha comprobado que las respuestas que dan los pacientes a las preguntas que se les hacen de cara a rellenar un DVA están influidas en gran medida por la respuesta inherente por defecto en la propia pregunta, lo que cuestiona la supuesta efectividad de los DVA para preservar la autonomía del paciente ${ }^{96}$.

A pesar de que las decisiones por sustituto son predictores imperfectos de los deseos de los pacientes ${ }^{97-99}$, muchos pacientes prefieren dejar estas decisiones en manos de sus seres queridos que escritas en un papel ${ }^{100,101}$. Pero tampoco es siempre así. En otro estudio se ha demostrado que aunque el $24 \%$ había hablado con su pareja del tema, el $29 \%$ no le nombró como sustituto porque considera que no es adecuado dejarle una carga así o porque "es demasiado emocional y no defendería lo que yo quiero" ${ }^{102}$. En caso de discrepancia entre paciente y sustituto ${ }^{103}$, el $46 \%$ prefería que se siguiera su propia decisión, el 57\% por motivos de respeto a la autonomía, el $18 \%$ por la dureza de la decisión para el sustituto, el $15 \%$ por considerar al sustituto demasiado emocional, y el $11 \%$ por conflicto de valores o de creencias con el sustituto. El $54 \%$ que prefería se siguiera la decisión tomada por el sustituto lo hacía por su confianza en la capacidad y el conocimiento del sustituto en un $63 \%$, por la relación de confianza con él en un $22 \%$, y por la preocupación por el mejor interés del sustituto en un 21\%. Puede decirse, pues, que para los pacientes sus relaciones son más importantes que las decisiones concretas, y que hacer descansar estas decisiones en personas importantes para el enfermo proporciona una sensación de control mayor que la propia toma de decisiones ${ }^{104}$. Esto parece confirmarse por los resultados de un estudio con pacientes con esclerosis lateral amiotrófica en el que se comprueba que entre un 53$70 \%$ había hablado con sus familiares sobre limitación de esfuerzo terapéutico, y que sólo un $26-34 \%$ lo había hecho con su médico $^{105}$.

Teno y col $^{106}$ analizan la efectividad práctica de la PSDA y dicen que "Los estilos habituales de práctica indican que el incre- mento de la existencia de Directivas Anticipadas no constituye un factor sustancial en la mejora del cuidado de los pacientes gravemente enfermos. Los futuros esfuerzos para mejorar la toma de decisiones deberían centrarse en los estilos habituales de práctica a través de una mayor comunicación y una planificación de la atención sanitaria más global".

En esa dirección se inscriben también las recomendaciones de Hickman et al. ${ }^{107}$ quienes afirman que se debe ir más allá de los DVA clásicos escritos que no han alcanzado su objetivo y que, en todo caso, éstos no debieran ser más que el final de un proceso de comunicación entre todos los participantes en la relación clínica, y que su objetivo sería el respeto al proyecto vital de cada uno en todas sus dimensiones.

\section{REFLEXIONES FINALES A MODO DE CONCLUSIONES}

La comunicación y la deliberación con los pacientes y sus seres queridos constituyen muchas veces uno de los aspectos más agradecidos del cuidado para muchas personas que van a dejar de vivir. Al final, son estas realidades últimas las que pueden llevarnos a vivir la muerte como un proceso natural y como una experiencia llena de sentido ${ }^{108}$.

Una razón importante del fracaso inicial de la puesta en marcha de los DVA reside en que no se ha dado la importancia que merece a la comunicación con los pacientes ${ }^{109,110}$ porque su propósito fundamental es el de ayudar a los pacientes a prepararse para la incapacidad y también para la muerte y no otros.

Deberíamos, por tanto, relegar los DVA al papel que deben tener, esto es, a ser una parte necesaria pero no fundamental de la PA. No hacerlo así es persistir en el error, reforzando el triunfo del dogma sobre la reflexión y de la esperanza sobre la experiencia ${ }^{111}$. Para ello se debe: 1 . Tratar la PA como un proceso permanente dentro de la relación clínica, y no como un acto dirigido a producir un producto, la firma de un documento; 2. Cambiar el centro de la PA hacia la facilitación de la deliberación sobre valores y preferencias; 3 . Llevar el centro de deliberación a la comunidad y a 
la unidad familiar; y 4. Promover la formación de los PS sobre los problemas del final de la vida.

Estas son las lecciones que se desprenden de las experiencias positivas con la PA de La Crosse en Winsconsin (EEUU) ${ }^{112}$ y de la Universidad de McMaster en Ontario (Canadá $)^{113}$. Pero poco o casi nada de esto se está haciendo en España (con alguna interesantísima excepción en Andalucía y Murcia, fundamentalmente) por lo que se están repitiendo de forma mimética todos y cada uno de los errores que la implantación de los DVA supuso en EEUU. Como si la experiencia de los demás no nos sirviera para nada.

Los DVA y las leyes que los regulan pueden ser interpretados literalmente, pero no basta con dar información a los pacientes ni con aceptar como válidos para siempre los deseos expresados en un momento concreto. Es cuando están sanos cuando los pacientes deben comenzar a hablar con sus seres queridos y los médicos sobre los $\mathrm{DVA}^{114}$. Y se debe deliberar no sólo sobre cómo quieren ser tratados en el futuro sino sobre sus valores, esperanzas, deseos e ilusiones. Y no sólo una vez, sino de forma mantenida a lo largo del tiempo.

Las personas, en tanto que seres sociales, tienen obligaciones mutuas. La autonomía no se da en el vacío sino en la vida en común. Las personas no lo somos fuera de los lazos sociales o de la comunidad, y ésta no puede existir, desarrollarse y crecer, sin la ayuda de las personas que la conforman. $\mathrm{Y}$ al tomar decisiones para uno, tenemos que ser conscientes de esta interdependencia ${ }^{115}$.

La PA no viene a complicarnos el trabajo. Todo lo contrario: la PA no es más que el reflejo de la complejidad de la situación en que ya nos movemos ${ }^{116}$. Cualquier situación problemática -y el final de la vida lo es- requiere medidas complejas, reflexión y diálogo. Por ello abogo y desde aquí animo a los PS y a las instituciones sanitarias a tener en cuenta esta interpretación del derecho a decidir y a poner en marcha cuantas medidas sean necesarias para hacerlo efectivo. En profundidad, porque profundas son las consecuencias de estas decisiones, para cada uno y para la sociedad.

\section{BIBLIOGRAFÍA}

1 Charles C, Gafni A, Whelan T. How to improve communication between doctors and patients. BMJ 2000; 320: 1220-1221.

2. Benbassat J, Pilpel D, Tidhar M. Patients' preferences for participation in clinical decision making: a review of published surveys. Behav Med 1998; 24: 81-88.

3. BALINT JA. Brief encounters: speaking with patients. Ann Intern Med 1999; 131: 231-234.

4. Martínez UrionabarRenetXeA K. Hacia un nuevo modelo de relación clínica. SEMERGEN: 2001; 27: 291-296.

5. Locke EA, Shaw K, SAari L, Latham GP. Goal setting and task performance. Psychol Bull 1981; 90: 125-152.

6. Austin J, VAnCOUVER J. Goals constructs in psychology: structure, process, and content. Psychol Bull 1996; 120: 338-375.

7. Muller JH. Shades of blue: the negotiation of limited codes by medical residents. Soc Sci Med 1992; 34: 885-892.

8. Fuchs V. The growing demand for medical care. N Engl J Med 1968; 179: 190-195.

9. Fox M, Lipton HL. The decision to perform cardiopulmonary resuscitation. N Engl J Med 1983; 309: 607-608.

10. Katz J. Why doctors don't disclose uncertainty. Hastings Cent Rep 1984; 14: 3544.

11. Bogardus ST, Bradley EH, Williams CS, MACIEJEWSKI PK, VAN DOORN C, INOUYE SK. Goals for the care of frail older adults: Do caregivers and clinicians agree? Am J Med 2001; 110: 670-673.

12. Levine C, Zuckerman C. The trouble with families: toward an ethic of acommodation. Ann Intern Med 1999; 130: 148-152.

13. Kutner L. Due process of euthanasia: the living will, a proposal. Indiana Law J 1969; 44: $537-574$

14. AnNaS GJ. Nancy Cruzan and the right to die. N Engl J Med 1990; 323: 670-673.

15. CRuZAN v. Director, Missouri Department of Health, 497 US 261 (1990).

16. Greco PJ, Schulman KA, Lavizzo-Mourey MR, Hansen-Flaschen J. The Patient SelfDetermination Act and the future of advance directives. Ann Intern Med 1991; 115: 639-643.

17. Singer PA, Siegler M. Advancing the cause of advance directives. Arch Intern Med 1992; 152: 22-24. 
18. Emanuel Ll, Barry MJ, Stoeckle JD, EtTelson LM, EmanuEl EJ. Advance directives for medical care: a case for greater use. N Engl J Med 1991; 324: 889-895.

19. Emanuel LL, Emanuel EJ. The medical directive. JAMA 1989; 261: 3288-3293.

20. Kelner MJ, Bougeault IL. Patient control over dying: responses of health care porfessionals. Soc Sci Med 1993; 36: 757765.

21. Lo B, Mcleod GA, Saika G. Patient attitudes to discussing life-sustaining treatment. Arch Intern Med 1986; 146: 1613-1615.

22. Shmerling RH, Bedell SE, Lilienfeld A, DELBANCO TL. Discussing cardiopulmonary resuscitation: a study of elderly outpatients. J Gen Intern Med 1988; 3: 317-321.

23. Frankl D, Oye RK, Bellamy PE. Attitudes of hospitalized patients toward life-support: a survey of 200 medical inpatients. Am J Med 1989; 86: 645-648.

24. Teno J, Leishman J, Brock DW, Mor V. The use of formal prior directives among patients with HIV-related disease. J Gen Intern Med 1990; 5: 490-494.

25. Stolman CJ, Gregory JJ, Dunn D, Levine JL. Evaluation of patient, physician, nurse and family attitudes toward do not resuscitate orders. Arch Intern Med 1991; 150: 653-658.

26. Gamble ER, McDonald PJ, Lichstein PR. Knowledge, attitudes and behaviour of elderly persons regarding living wills. Arch Intern Med 1991; 151: 277-280.

27. Joos SK, Reuler JB, Powell JL, Hickemn DH. Outpatients' attitudes and understanding regarding living wills. J Gen Intern Med 1993;8: 259-263.

28. Pfeifer MP, Sidorov JE, Smith AC, Boero JF, Evans AT, SETtLe MB. The discussion of endof-life medical care by primary care patients and their physicians: a multicenter study using structured qualitative interviews. J Gen Intern Med 1994; 9: 82-88.

29. Molloy DW, Guyatt G, Elemayheu E, Mcllroy WE. Treatment preferences, attitudes toward advance directives and concerns about health care. Humane Med 1991; 7: 285-290.

30. AnNAS GJ. The heatlh care proxy and the living will. N Engl J Med 1991; 324: 12101213.

31. BRETT AS. Limitations of listing specific medical interventions in advance directives. JAMA 1991; 226: 825-828.
32. OLICK RS. Aproximating informed consent and fostering communication. J Clin Ethics 1991; 2:181-195

33. Wolf SM, Boyle P, Callahan D, Fins J, Jennings B, NELSON B et al. Sources of concern about the Patient Self-Determination Act. N Engl J Med 1991; 325: 1666-1671.

34. Instrumento de ratificación del convenio para la protección de los derechos humanos y la dignidad del ser humano con respecto a las aplicaciones de la biología y la medicina (convenio relativo a los derechos humanos y la biomedicina). Oviedo, 4 de abril de 1997 . BOE n. ${ }^{\circ} 251$, de 20 de octubre de 1999.

35. Ley 41/2002, de 14 de noviembre, básica reguladora de la autonomía del paciente y de derechos y obligaciones en materia de información y documentación clínica. BOE n. ${ }^{\circ} 274$, de 15 de noviembre de 2002.

36. Simón P, BARRIo I. ¿Quién puede decidir por mi? Una revisión de la legislación española vigente sobre las decisiones de representación y las instrucciones previas. Rev Cal Asist 2004; 19: 460-472.

37. Blanco Zabala J. Autonomía e Instrucciones Previas. Un análisis comparativo de las legislaciones autonómicas del Estado Español. Tesis Doctoral, 2007.

38. SEOAnE JA. Derecho y planificación anticipada de la atención: panorama jurídico de las Intsrucciones Previas en España. DS Derecho y Salud 2006; 14: 285-295.

39. Couceiro VIDAL A. Las directivas anticipadas en España: contenido, límites y aplicaciones clínicas. Rev Cal Asist 2007; 22: 213-222.

40. Bachiller A, Hernández de Miguel S, Martínez M, Delgado R, Domínguez V. Testamento Vital: La opinión médica en la provincia de Valladolid. Metas Enferm 2004; 7: 24-27.

41. Zabala J, Alconero AR, Casaus M, Gutiérrez E, SAIz G. Evaluación de aspectos bioéticos en los profesionales de la salud. Enferm Clin 2007; 17: 56-62.

42. Asociación Nacional de Consumidores y Usuarios de Servicios de Salud (ASUSALUD). Análisis de situación y propuestas de mejora. La normativa sobre el "Testamento Vital", dispersa y poco conocida. [Monografía en Internet]. Disponible en http://www.asusalud.org/asusalud/ficheros/ ASU_114_f0.pdf.

43. Bosch JM. Documento de voluntades anticipadas (DVA): cinco años recorridos y mucho por avanzar. Sobre Ruedas 2006; 63: $2-5$. 
44. Miró G, Pedrol E, Soler A, Serra-Prat M, YÉBENES JC, MARTínEz R et al. Conocimientos de la enfermedad y de los documentos de voluntades anticipadas en el paciente seropositivo para VIH. Med Clin 2006; 126: 567572.

45. Solsona JF, Sucarrats A, Maull E, Barbat C, García S, Villares S. Toma de decisiones al final de la vida. Encuesta sobre la actitud ante la muerte del paciente crítico. Med Clin 2003; 120: 335-336.

46. BRETT AS: Limitations of listing medical interventions in advance directives. JAMA 1991; 266: 825-828

47. EPSTEIn RM. Mindful practice. JAMA 1999; 282: 833-839.

48. Silveira MJ, DiPiero A, Gerrity MS, Feudtner C. Patients' knowlegde of options at the end of life: Ignorance at the face of deatk JAMA 2000; 284: 2483, 2486-2487.

49. Yamada R, Galecki AT, Goold SD, Hogikyan RV. A multimedia intervention on cardiopulmonary resuscitation and advance directives. J Gen Intern Med 1999; 14: 559563.

50. Miles SH. Advance directives to limit treatment: The need for portability. J Am Geriats Soc 1987; 35: 74-76.

51. McNeil BJ, Pauker SJ, Sox HC, Tversky A. On the elicitation of preferences for alternative therapies. NEJM 1982; 306: 1259-1262.

52. Mazur DJ, HickMan DH. Patient preferences: Survival versus quality-of-life considerations. J G Intern Med 1993; 8: 3764-377.

53. MAZUR DJ, MERZ JF. How age, outcome severity, and scale influence general medicine clinic patients' interpretations of verbal probability terms. J Gen Intern Med 1994; 9: 268-271.

54. EISER AR, WeISS MD. The underachieving advance directive: Recommendations for increasing advance directive completion. Am J Bioethics 2001; 1: 1-15.

55. Virmani J, Schneiderman LJ, Kaplan RM. Relationship of advance directives to physician-patient communication. Arch Intern Med 1994; 154: 909-913.

56. Tulsky JA; Chesney MA, Lo B. How do medical residents discuss resuscitation with patients? J Gn Intern Med 1995; 10: 436-442.

57. Fischer GS, Tulsky JA, Rose MR, Siminoff LA, ARNOLD RM. Patient knowledge and physician predictions of treatment preferences after discussion of advance directives. J Gen Intern Med 1998; 13: 447-454.
58. Ditto PH, Danks JH, Smucker WD, Bookwala J, Coppola KM, DRESSER R et al. Advance directives as acts of communication: A randomized controlled trial. Arch Intern Med 2001; 161: 421-430.

59. EMANUEL L. Living wills can help doctors and patients talk about dying. W J Med 2000; 173: 368 .

60. WELIE JVM. Living wills and substituted judgements: a critical anlysis. Med Health Care and Philos 2001; 4: 169-183.

61. PARFITT D. Reasons and persons. Oxford: Oxford University Press, 1984.

62. DRESSER R. Missing persons: Legal perceptions of incompetent patients. Rutger Law Rev 1994; 46: 609-695.

63. DRESSER R. Dworkin on dementia. Elegant theory, questionable policy. Hastings Cent Rep 1995; 25: 32-38

64. Christenscn-Szalanski JJ. Discount functions and the measurement of patients' values: Women's decisions during childbirth. Med Dec Making 1984; 1: 47-58.

65. GREAdy RM, DitTo PH, DanKs JH, Coppola KM, LOCKHART LK. Actual and perceived stability of preferentes for life-sustaining treatment. J Clin Ethics 2000; 11: 334-346.

66. GILBERT DT, WILSON TD. Miswanting: Some problems in the forecasting of future affective status. En: Feeling and Thinking: The role of affect in social cognition, ed., JP Forgas (New York: Cambridge University Press, 2000).

67. Kahneman D, Snell J. Predicting a changing tatse: Do people know what they will like? J Behav Dec Making 1992; 1: 187-200.

68. Loewenstcin G, SchKade D. Wouldn't it be nice? Predicting future feelings. En: Hedonic Pscyhology: Scientific approaches to enjoyment, suffering and wellbeing, ed., N Schawrtz y D Kahneman (New York: Russel Sage Foundation, 1997).

69. SCHNEIDER CE. The practice of autonomy: Patients, doctors, and medical decisions. New York: Oxford University Press, 1998.

70. Smith ML, Gremillion G, Slomka J, Warneke CL. Texas hospitals' experience with the Texas Advance Directives Act. Crit Care Med 2007; 35: 1271-1276.

71. Truog RD. Tackling medical futility in Texas. NEJM 2007; 357: 1-3.

72. Verheijde JL, Rady MY, McGregor JL. The United States Revised Uniform Anatomical Gift Act (2006): New challenges to balancing patient rights and physician 
responsibilities. Philos Ethics Humanit Med 2007; 2: 19-

73. BROCK D. Trumping advance directives. Hastings Cent Rep 1991; 21: S5-S6.

74. La Puma J, Orentlicher D, Moss RJ. Advance Directives on admission. Clinical implications and analysis of the Patient Self-Determination Act of 1990. JAMA 1991; 266: 402-405.

75. Cox DM, SAchs GA. Advance directives and the PSDA. Clin Geriatr Med 1994; 10: 431443.

76. Miles SH, Koepr R, Weber EP. Advance nedof-life treatment planning: A research review. Arch Intern Med 1996;156: 10621068.

77. HigH DM. All in the family: Extended autonomy and expectations in surrogate heatl care decisión-making. Gerontologist 1988; 28S: 46-51.

78. RoE JM. Durable power of attorney for health care: A survey of senior center participants. Arch Int Med 1992; 152: 292296.

79. Sugarman J, Weinberger M, Samsa G. Factors associated with Veternas decisions about living wills. Arch Inte Med 1992; 152: 343347.

80. Santos de Unamuno C. Documento de Voluntades Anticipadas: actitud de los pacientes de atención primaria. Aten Primaria 2003; 32: $30-35$.

81. HaHn ME. Advance Directives and PatientPhysician communication. JAMA 2003; 289:96.

82. Tierney WM, DeXter PR, Gramelspacher GP, Perkins AJ, ZHou XH, Wolinsky FD. The effect of discussion about advance directives on patients' satisfaction with primary care. J Gen Intern Med 2001; 16: 32-40.

83. DAVISON SN. Facilitating advance care planning for patients with end-stage renal disease: the patient perspective. Clin J Am Soc Nephrol 2006; 1: 1023-1028.

84. Jezewski MA, Meeker MA, Sessana L, Finnel D. The effectiveness of interventions to increase Advance Directives completion. J Aging Health 2007; 19: 519-536.

85. Brown JB, Beck A, Boles M, Barreto P. Practical methods to increase use of advance medical directives. J Gen Intern Med 1999; 14: $21-26$

86. Busquets JM, VÁllez C. Consideracions entorn les voluntats anticipades. Annals de Medicina [Revista en Internet] 2003 abril / maig / juny; 86 (2). Disponible en http://www.academia.cat/cas/pages/acade $\mathrm{m} /$ vidaacad/publica/annals/annals_f.htm.

87. BETANCOR JT. La voluntat anticipada: reflexions desde l'experiència associativa. Annals de Medicina [Revista en Internet] 2003 abril / maig / juny; 86 (2). Disponible en http://www.academia.cat/cas/pages/ academ/vidaacad/publica/annals/annals_f. htm.

88. TERribas N. Un documento de voluntades anticipadas. FMC Form Med Contin Aten Prim 2006; 13: 354-357.

89. Martínez URIONABARRENETXEA K. Reflexiones sobre el testamento vital. Aten Prim 2003; 31: $52-54$

90. BARRIo IM, Simón P, PASCAU MJ. El papel de la enfermera en la planificación anticipada de las decisiones: más allá de las instrucciones previas o voluntades anticipadas. Enferm Clin 2004; 14: 223-229.

91. Abad E, García A, Martínez S, Sánchez R, Molina A. Exploración del fenómeno de la planificación anticipada de decisiones al final de la vida. Visión de los profesionales que cuidan personas mayores. Enferm Clin 2006; 16: 127-136.

92. Arranz P, Barbero JJ, Barreto P, Bayés R. Intervención emocional en cuidados paliativos. Modelos y protocolos. Barcelona: Ariel, 2003.

93. Drought TS, Koenig BA, Raffin TA. Advance directives: Changing our expectations. Chest 1996; 110: 589-591.

94. TAUBER AI. Patient autonomy and the ethics of responsibility. Cambridge, MA: MIT Press, 2005.

95. Singer PA, Robertson G, Roy DJ, Theol PL. Advance care planning. Can Med Assoc J 1996; 155: 1689-1692.

96. PERKInS HS. Controlling death: The false promise of advance directives. Ann Intern Med 2007; 147: 51-57.

97. Emanuel LL, von Gunten CF, Ferris FD. Advance care planning. Arch Fam Med 2000; 9: 1181-1187.

98. Teno JM, Lindemann H, Lynn J. Advance care planning. Priorities for ethical and empirical research. Hastings Cent Rep Nov-Dec 1994; S32-S36.

99. LAMBert PG, Gibson JM, Nathanson P. The values history: an innovation in surrogate medical decision making. Law Med Health Care 1990; 3: 202-212.

100. Doukas DJ, McCullough LB. The values history: the evaluation of patients' values 
and advance directives. J Fam Pract 1991; 32: 145-153.

101. Doukas DJ, Antonucci TA, Gorenflo DW. Multigenerational study on the correlation of values and advance directives. Ethics and Behav 1992; 2: 51-59.

102. Kolarik RC, ARnold RM, Fischer GS, Haunsa $\mathrm{BH}$. Advance care planning. A comparison of values statement and treatment preferences. J Gen Intern Med 2002; 17: 618624.

103. Martin DK, Emanuel LL, Singer PA. Planning for the end of life. Lancet 2000; 356: 16721676.

104. Teno JM, LynN J. Putting advance-care planning into action. J Clin Ethics 1996; 7: 205-213.

105. LARSON DG, TOBIN DR. End-of-life conversations: evolving theory and practice. JAMA 2000; 284: 1573-1578.

106. LAINE C, DAVIDOFF F. Patient-centered medicine. A professional evolution. JAMA 1996; 275: 152-156.

107. Danis M, Garret J, Harris R, PATrick DL. Stability of choices about life-sustaining treatment. Ann Intern Med 1994; 120: 567573.

108. Emanuel LL, Emanuel EJ, Stoeckle JD, Hummel LR, BARRY MJ. Advance directives: stability of patients' treatment choices. Arch Intern Med 1994; 154: 209-217.

109. GREAdy RM, DitTo PH, DANKS JH, Coppola KM, LOCKHART LK, SMUCKER WD. Acvtual and perceived stability of preferences for lifesustaining treatment. J Clin Ethics 2000; 11: 334-346.

110. Malloy TR, Wigton RS, Meeske J, Tape TG. The influence of treatment descriptions on advance medical directive decisions. J Am Geriatr Soc 1992; 40: 1255-1260.

111. Sehgal A, Galbraith A, Chesney M, Schonfeld P, Charles G, Lo B. How strictly do dyalisis patients want their advance directives followed? JAMA 1992; 267: 59-63.

112. Kressel LM, Chapman GB, Leventhal E. The influence of default options on the expressiojn of end-of-life treatment preferences in advance directives. J Gen Intern Med 2007; 22: 1007-1010.

113. Sulmasy DP, Terry PB, Weisman CS, Miller DJ, StAlling RY, Vettese MA et al. The accuracy of substituted judgements in patients with terminal diagnoses. Ann Intern Med 1998; 128: 621-629.
114. Suhl J, Simons P, ReEdy T, Garrick T. Myth of substituted judgement. Arch Intern Med 1994; 154: 90-96.

115. Hare J, Pratt C, Nelson C. Agreement between patients and their self-selected surrogates on difficult medical decisions. Arch Intern Med 1992; 152: 1049-1054.

116. Seckler AB, Meier DE, Mulvihill M, Paris BE. Substituted judgement: how accurate are prowy predictions?. Ann Intern Med 1991; 115: $92-98$

117. PuChalski CM, Zhong A, Jacobs MM, LynN J, Harrold J, Galanos A et al. Patients who want their family and physician to make resuscitation decisions for them: observations from SUPPORT and HELP. J Am Geriatr Soc 2000; 48 (S5): S84-S90.

118. HIGH DM. Why are elderly people not using advance directives?. J Am Geriatr Soc 1993; 5: 497-515.

119. SchifF R, RAJKUMAR C, BulpitT C. Views of elderly people on living wills: interview study. BMJ 2000; 320: 1640-1641.

120. Terry PB, Vettsse M, Song J, Forman J, Haller KB, Miller DJ et al. End-of-life decision making: when patients and surrogates disagree. J Clin Ethics 1999; 10: 286-293.

121. HANSON LC, Danis M, GARRETT J. What is wrong with end-of-life care?. Opinions of bereaved family members. J Am Geriatr Soc 1997; 45: 1339-1344.

122. Moss AH, Oppenheimer EA, Casey P, Cazzolli PA, Roos RP, Stocking CB, Siegler M. Patients with amyotrophic lateral sclerosis receiving long-term mechanical ventilation. Advance care planning and outcomes. Chest 1996; 110: 249-255.

123. Teno JM, Lynn J, Wenger N, Phillips RS, Murphy $\mathrm{DP}$ et al. Advance directives for seriously ill hospitalized patients: effectiveness with the Patient Self-Determination Act and the SUPPORT intervention. J Am Geriatr Soc 1997; 45: 500-507.

124. Hickman SE, Hammes BJ, Moss AH, Tolle SW. Hope for the future: Achieving the original intent of Advance Directives. Hastings Cen Rep 2005; Nov/Dec: 26-30.

125. MCCUE JD. The naturalness of dying. JAMA 1989; 87: 81-84

126. Singer PA, Martin DK, LaVERy J, Thiel EC, Kelner M, MENDELSSOHN DC. Reconceptualizing advance care planning from the patient's perspective. Arch Intern Med 1998; 158: 879-884. 
127. Martin DK, Thiel EC, Singer PA. A new model of advance care planning: observations from people with HIV. Arch Intern Med 1999; 159: 86-92.

128. Fagerlin A, Schneider C. The failure of the living will. Hastings Cent Rep 2004; 32: 3042.

129. Hammes BJ, Rooney BL. Death and end-of-life planning in one midwestern community. Arch Intern Med 1998; 158: 383-390.

30. Molloy DW, Guyatt GH, Russo R, Goeree R, O'Brien BJ, BEdARD M et al. Systematic implementation of an advance directive program in nursing homes. JAMA 2000; 283: 1437-1444.
130. Molloy DW, Guyatt GH, Russo R, Goeree R, O'BRIEN BJ, BEDARD $M$ et al. Systematic implementation of an advance directive program in nursing homes. JAMA 2000; 283 : 1437-1444.

131. Wissow LS, Belote A, Kramer W, ComptonPhillips A, KRITZler R, Weiner JP. Promoting advance directives among elderly primary care patients. J Gen Intern Med 2004; 19 : 944-951.

132. LoEwY EH. Freedom and community: the ethics of interdependence. Albany, NY: Suny Press, 1993.

133. Broggi MA. El documento de voluntades anticipadas. Med Clin 2001; 117: 114-115. 